\title{
Nonequilibrium Floquet Steady States of Time-Periodic Driven Luttinger Liquids
}

\author{
Serena Fazzini, ${ }^{1}$ Piotr Chudzinski, ${ }^{2,3}$ Christoph Dauer, ${ }^{1}$ Imke Schneider, ${ }^{1,4}$ and Sebastian Eggert ${ }^{1}$ \\ ${ }^{1}$ Physics Department and Research Center OPTIMAS, Technische Universität Kaiserslautern, \\ 67663 Kaiserslautern, Germany \\ ${ }^{2}$ School of Mathematics and Physics, Queen's University of Belfast, BT7 1NN Belfast, United Kingdom \\ ${ }^{3}$ Institute of Fundamental Technological Research, Polish Academy of Science, 02-106 Warszawa, Poland \\ ${ }^{4}$ Institute of Physics, Universität Augsburg, 86135 Augsburg, Germany
}

(Received 16 November 2020; revised 8 March 2021; accepted 20 May 2021; published 17 June 2021)

\begin{abstract}
Time-periodic driving facilitates a wealth of novel quantum states and quantum engineering. The interplay of Floquet states and strong interactions is particularly intriguing, which we study using timeperiodic fields in a one-dimensional quantum gas, modeled by a Luttinger liquid with periodically changing interactions. By developing a time-periodic operator algebra, we are able to solve and analyze the complete set of nonequilibrium steady states in terms of a Floquet-Bogoliubov ansatz and known analytic functions. Complex valued Floquet eigenenergies occur when integer multiples of the driving frequency approximately match twice the dispersion energy, which correspond to resonant states. In experimental systems of Lieb-Liniger bosons we predict a change from power-law correlations to dominant collective density wave excitations at the corresponding wave numbers as the frequency is lowered below a characteristic cutoff.
\end{abstract}

DOI: 10.1103/PhysRevLett.126.243401

Introduction.-Controlled time-periodic driving of quantum systems has recently pushed the development of fascinating quantum phenomena such as topological phases [1,2], many body localization [3], cavity optomechanics [4-12], Floquet time crystals [13,14], artificial gauge fields [15-20], transmission resonances [21-23], dynamic localization [24-29], pairing [30,31], driven Bose-Einstein condensates [32-39], and anyons [40-44]. However, when complications from strong correlations and nonequilibrium physics become intertwined, understanding the dynamics becomes very difficult. Theoretical progress has been made in the high frequency limit [45-47], which is useful for Floquet engineering. On the other hand, it is extremely rare to obtain full solutions of time-periodically driven manybody systems, which could give much needed insight in Floquet-induced strong correlations near resonances.

In this Letter we now provide the many-body eigenstate solution and report resonance phenomena in onedimensional (1D) interacting quantum systems with time-periodically driven parameters. Our analysis applies to time-periodic driving of generic Tomonaga-Luttinger liquids (TLL), which describe a large class of effectively

Published by the American Physical Society under the terms of the Creative Commons Attribution 4.0 International license. Further distribution of this work must maintain attribution to the author(s) and the published article's title, journal citation, and DOI.
1D many-body systems [48] and can also be realized using ultracold gases [49-52]. The time-evolution of an initially prepared state in a TLL has been calculated before [53-60], but much less is known about the nature of possible nonequilibrium steady states under periodic driving. It is therefore desirable to obtain the full eigenbasis of the Floquet eigenvalue problem, which gives systematic information about all stable steady states and their corresponding dominant correlations. We now obtain the explicit steady state solutions of the time-dependent Schrödinger equation of a quantum many-body system in terms of a time-periodic operator algebra, which not only allow a full analysis using closed analytic functions but also show regions of instabilities in frequency and momentum space. We therefore predict large-amplitude density waves at the characteristic wave vectors in trapped ultracold boson systems.

Model.-We will develop a Floquet-Bogoliubov ansatz for general driven TLL models. To make concrete predictions for decoupled 1D tubes of interacting bosons in optical lattices [49-52] we choose the Lieb-Liniger Hamiltonian [61] as a starting point

$$
H_{0}=-\frac{1}{2 m} \sum_{i=1}^{N} \frac{\partial^{2}}{\partial x_{i}^{2}}+g \sum_{i<j=1}^{N} \delta\left(x_{i}-x_{j}\right)
$$

where $\hbar=1$ and $g=2 a_{0} /\left[m a_{\perp}\left(a_{\perp}-1.03 a_{0}\right)\right]$ is the $1 \mathrm{D}$ onsite interaction strength, which is tunable via the $3 \mathrm{D}$ scattering length $a_{0}$ and the perpendicular confinement length $a_{\perp}$ [62-65]. The static system is integrable and 
correlations are known to be well described by a TLL model in the long-wavelength limit $q<q_{c}[48,63,64]$

$$
\begin{aligned}
H_{T L L} & =\frac{v}{2 \pi} \int d x\left[K\left(\partial_{x} \theta\right)^{2}+K^{-1}\left(\partial_{x} \phi\right)^{2}\right]=\sum_{q>0} H_{q}, \\
H_{q} & =v_{F} q\left[\left(1+g_{4}\right) 2 J_{0, q}+g_{2}\left(J_{+, q}+J_{-, q}\right)\right]
\end{aligned}
$$

where $2 J_{0, q}=b_{L, q}^{\dagger} b_{L, q}+b_{R, q} b_{R, q}^{\dagger} \quad$ and $\quad J_{+, q}=J_{-, q}^{\dagger}=$ $b_{L, q}^{\dagger} b_{R, q}^{\dagger}$ are SU(1,1) generators [66-68] in terms of bosonic operators $b_{L / R, q}^{\dagger}$, which create left- and right-moving density waves at wave-vector $q$ [48]. For the LiebLininger model the Luttinger parameter $K$ is known exactly [63-65]. It depends only on the ratio $m g / n$, where $n$ is the 1D particle density. The cutoff $q_{c}$ above which the TLL description fails has also been determined [64]. The scattering amplitudes $g_{2}$ and $g_{4}$ are rescaled from the traditional " $g$-ology" scheme [69] and related to $v$ and $K$ by [48]

$v K=v_{F}\left(1+g_{4}-g_{2}\right), \quad \frac{v}{K}=v_{F}\left(1+g_{4}+g_{2}\right)$,

where $v_{F}=\pi n / m$. Values of $g_{2}=g_{4}=\left(1 / K^{2}-1\right) / 2$ for the Lieb-Liniger model are shown in Fig. 1.

We now turn to systems with time-periodically changing control parameters $a_{0}$ and $a_{\perp}$, which will result in timeperiodic couplings $g, g_{2}$, and $g_{4}$, all of which can be determined exactly. Any desired time-periodic couplings can be created by suitable fields given by the inverted relations, including a pure sinusoidal behavior [70]

$$
2 g_{2}(t)=2 g_{4}(t)=\bar{\rho}+\rho \cos \omega t
$$

with constant parameters $\bar{\rho}$ and $\rho$. We will later consider more general behavior. For the Lieb-Liniger model it is known that $v K=v_{F}$ and $K>1[63,64]$, so that from Eq. (3) $-1 / 2<g_{2}=g_{4}<0$ as shown in Fig. 1.

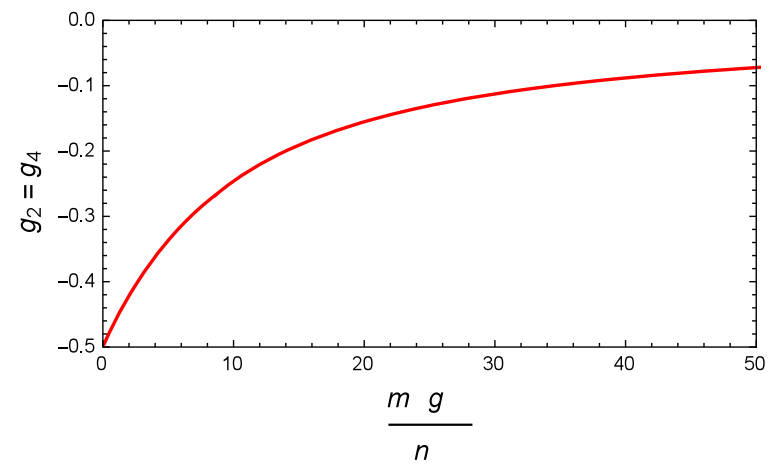

FIG. 1. Coupling constants $g_{2}=g_{4}=\left(1 / K^{2}-1\right) / 2$ for the Lieb-Liniger model as a function of $m g / n$ [63-65], which can be determined for any value of $a_{0}$ and $a_{\perp}$.
Floquet ansatz.-We now seek to solve the timedependent Schrödinger equation $\imath \partial_{t}|\Psi(t)\rangle=H_{q}(t)|\Psi(t)\rangle$ for each momentum $q$ separately, which remains a good quantum number and can be omitted in the following. According to Floquet theory $[27,46,47,71]$ there exists a complete set of quantum numbers $n$ for steady state solutions $\left|\Psi_{n}(t)\right\rangle=e^{-l \epsilon_{n} t}\left|u_{n}(t)\right\rangle$. Here $\left|u_{n}(t)\right\rangle=\left|u_{n}(t+T)\right\rangle$ with $T=2 \pi / \omega$ obey the Floquet equation

$$
\left[H(t)-\imath \partial_{t}\right]\left|u_{n}(t)\right\rangle=\epsilon_{n}\left|u_{n}(t)\right\rangle
$$

where $\epsilon_{n}$ are the Floquet quasienergies. We now wish to map this problem onto a static eigenvalue problem [72]

$$
\tilde{H}|n\rangle=\left(Q H Q^{\dagger}-i Q \partial_{t} Q^{\dagger}\right)|n\rangle=\epsilon_{n}|n\rangle .
$$

Floquet theory has been reviewed extensively [27,46, 47,71], but the ansatz (6) goes beyond the usual timeevolution approach since it makes the problem static, diagonalizes it in the original Hilbert space, and determines all steady states for all times in one single unitary transformation $Q(t)$, which is an ambitious goal. The relation of $Q=\sum_{n}|n\rangle\left\langle u_{n}(t)\right|$ to Floquet concepts is discussed in the Supplemental Material [73]: While the time-evolution operator $W(t)$ is not the topic here, it can be simply obtained $W(t)=Q^{\dagger}(t) e^{-i \tilde{H} t} Q(0)$. However, it is not possible to construct $Q$ using $W$. Likewise, the so-called Floquet Hamiltonian [27,46,47,71] $H_{F}=Q^{\dagger}(0) \tilde{H} Q(0)$ can be found using $Q$. We now proceed to find an explicit expression for $Q(t)$ for the model in Eq. (2).

Floquet Bogoliubov solution.-The goal is to find a static eigenbasis in the rotating frame, which can be achieved if $\tilde{H}$ becomes diagonal and time independent. The interacting model $H_{q}(t)$ in Eq. (2) is defined in left and right oscillator Hilbert spaces $\chi=L, R$, so a static solution must be of the form $\tilde{H}=\Delta \sum_{\chi} b_{\chi}^{\dagger} b_{\chi}$. The characteristic commutation relation $\left[\tilde{H}, b_{L, R}\right]=-\Delta b_{L, R}$ transforms to

$$
\begin{gathered}
{\left[\left(H(t)-\imath \partial_{t}\right), \beta_{L, R}(t)\right]=-\Delta \beta_{L, R}(t) \quad \text { with }} \\
\beta_{L, R}(t)=Q^{\dagger}(t) b_{L, R} Q(t)=\gamma_{1}(t) b_{L, R}+\gamma_{2}(t) b_{R, L}^{\dagger}
\end{gathered}
$$

where we have used a general Floquet-Bogoliubov ansatz for $Q$ in Eq. (8) with the canonical constraint $\left|\gamma_{1}(t)\right|^{2}-\left|\gamma_{2}(t)\right|^{2}=1$. The defining relation in Eq. (7) provides differential equations for the time-periodic coefficients $\gamma_{1,2}$

$$
\dot{\gamma}_{1,2}=\left(\Delta \mp \lambda_{1}\right) \gamma_{1,2} \pm \lambda_{2} \gamma_{2,1}
$$

where $\lambda_{1}=v_{F} q\left(1+g_{4}\right)$ and $\lambda_{2}=v_{F} q g_{2}$. The relation (9) applies to general TLL, but for the Lieb-Liniger model it simplifies since $\lambda_{1}-\lambda_{2}=q v_{F}$ is constant due to Galilean 
invariance. Using $f_{ \pm}(t)=e^{\imath \Delta t}\left[\gamma_{1}(t) \pm \gamma_{2}(t)\right]$ and Eq. (4) we obtain a Mathieu equation

$$
\ddot{f}_{-}(t)+q^{2} v_{F}^{2}(1+\bar{\rho}+\rho \cos \omega t) f_{-}(t)=0
$$

and $f_{+}=-i \dot{f}_{-} / q v_{F}$. The solution can be expressed as

$$
\begin{aligned}
f_{-}(t) & =c_{1} \mathcal{C}(a, p, \tau)+c_{2} \mathcal{S}(a, p, \tau) \\
\text { where } a & =4 \frac{q^{2} v_{F}^{2}}{\omega^{2}}(1+\bar{\rho}), \quad p=-2 \frac{q^{2} v_{F}^{2}}{\omega^{2}} \rho, \quad \tau=\frac{\omega t}{2},
\end{aligned}
$$

and $\mathcal{C}(a, p, \tau), \mathcal{S}(a, p, \tau)$ are even and odd Mathieu functions normalized with $\mathcal{C}(a, p, 0)=\mathcal{S}(a, p, \pi)=1$. The coefficients $c_{1,2}$ are determined by the time periodicity of steady states $\left|u_{n}(t)\right\rangle$ and operators $\beta(t)$, which also fixes the quantization condition for $\Delta$ : We use Floquet's theorem to write the solution of Eq. (10) $f_{-}(t)=e^{i \nu \tau} P_{\nu}(\tau)$ with $P_{\nu}(\tau)=P_{\nu}(\tau+\pi)$ [74]. Since $\gamma_{1 / 2}$ are periodic, we find that the Mathieu characteristic exponent is $\nu=2 \Delta / \omega$, which must be real for stable steady states, just like for the Mathieu stability chart [75] of Paul traps [76]. From the normalization above follows $\cos (\pi \nu)=\mathcal{C}[a, p, \pi]$, which gives [73]

$$
\Delta=\arccos [\mathcal{C}(a, p, \pi)] / T, \quad c_{2}=\imath c_{1} \sin T \Delta,
$$

and $c_{1}$ is fixed by $\left|\gamma_{1}\right|^{2}-\left|\gamma_{2}\right|^{2}=1$. Last but not least, we can use the solutions of $\gamma_{1,2}$ to uniquely define three real time periodic functions $\theta, \phi, r$, which parametrize an explicit expression of $Q(t)$ in Eq. (8) in terms of the $\mathrm{SU}(1,1)$ generators $J_{0}, J_{-}$, and $J_{+}$in Eq. (2) [66-68]

$$
\begin{gathered}
Q(t)=e^{i \theta J_{0}} e^{r\left(J_{+}-J_{-}\right)} e^{-i \phi J_{0}} \quad \text { with } \\
\gamma_{1}=e^{i(\theta-\phi) / 2} \cosh r, \quad \gamma_{2}=e^{i(\theta+\phi) / 2} \sinh r .
\end{gathered}
$$

In the Supplemental Material [73] it is shown that $Q(t)$ in Eq. (13) gives $\tilde{H}=\Delta\left(b_{L}^{\dagger} b_{L}+b_{R} b_{R}^{\dagger}\right)$ and the form of the transformed ground state $\left|u_{0}(t)\right\rangle=Q^{\dagger}|0\rangle$ is provided [73], which obeys $\beta_{L, R}(t)\left|u_{0}(t)\right\rangle=0 \forall t$. Therefore, from Eqs. (7) and (8) all Floquet modes $\left|u_{n}(t)\right\rangle$ with $\epsilon_{n}=\left(n_{L}+n_{R}+1\right) \Delta$ are found by application of $\left[\beta_{L}^{\dagger}(t)\right]^{n_{L}}\left[\beta_{R}^{\dagger}(t)\right]^{n_{R}}$ on $\left|u_{0}(t)\right\rangle$.

Instability regions.-Before calculating physical observables, we need to analyze the stability of the differential equations, which may not always have a solution due to the periodicity constraint. In Fig. 2 (top) we plot the value of $\nu=2 \Delta / \omega$ as a function of rescaled momenta $q v_{F} / \omega$ using $\bar{\rho}=-0.6$ and amplitude $\rho=0.25$. We observe that for certain regions of momenta there are no real solutions for $\Delta$. These "instability regions" will have interesting physical implications as discussed below. The stable regions are shown as a function of amplitude $\rho$ in
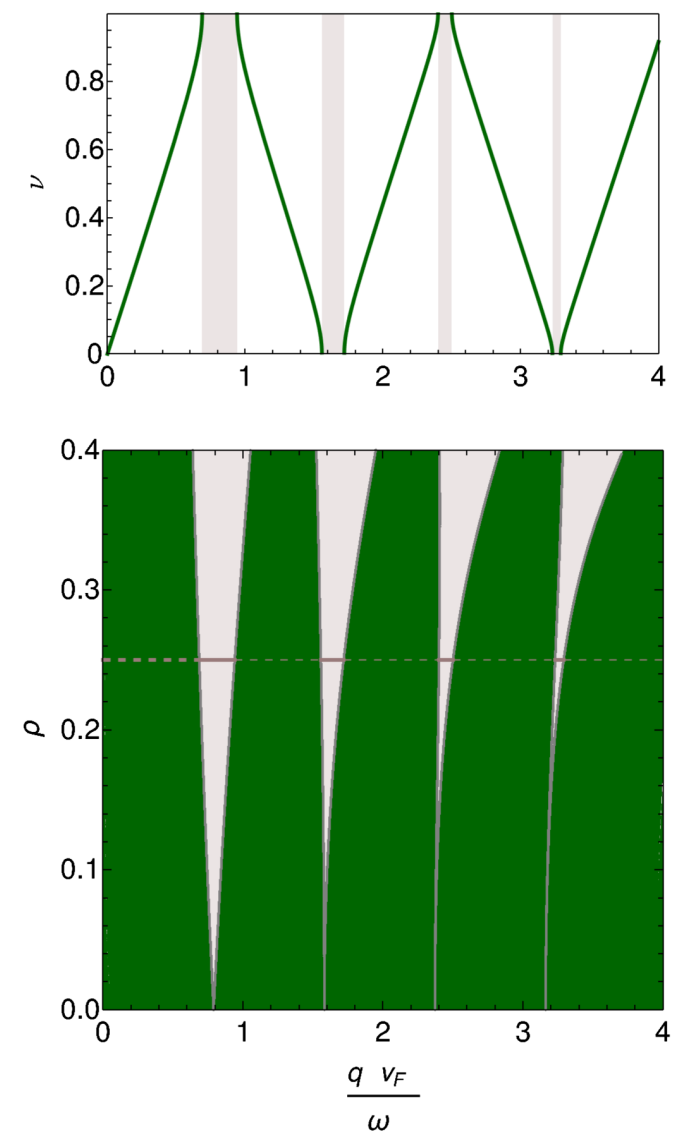

FIG. 2. Top: value of $\nu=2 \Delta / \omega$ as a function of $q v_{F} / \omega$ using $\bar{\rho}=-0.6$ and amplitude $\rho=0.25$. Shaded regions indicate complex values of $\Delta$. Bottom: stability chart of the Mathieu equation with $\bar{\rho}=-0.6$. Grey areas are the instability regions around the resonance points $q_{\ell}=\ell \omega / 2 \bar{v}$.

Fig. 2 (bottom) for $\bar{\rho}=-0.6$. For small $\rho$ the instability regions are equally spaced at integer values $\ell \in \mathbb{N}$ corresponding to $a=\ell^{2}$ or $\ell=2 \sqrt{1+\bar{\rho}} q v_{F} / \omega$. Defining an average velocity $\bar{v}=v_{F} / \bar{K}=v_{F} \sqrt{1+\bar{\rho}}$ the instability regions therefore correspond to integer multiples of frequency which match twice the interacting dispersion relation $\ell \omega=2 \bar{v} q_{\ell}$, so the physical cause can be traced to resonant excitations on the linear branches of left movers from $-\bar{v} q_{\ell}$ to right movers at $\bar{v} q_{\ell}$ and vice versa.

As shown in Fig. 3, the region of instabilities also occurs for more general TLL models where the restriction $g_{2}(t)=g_{4}(t)$ in Eq. (4) is lifted [60] and/or contains higher harmonics. A general analytic solution remains elusive, but the corresponding differential equation (9) is still valid, which we have solved numerically by Fourier decomposition for several parameters. Instability regions are always expected since the problem is analogous to forbidden energy regions in a band structure of a periodic potential [71], which is of course generic. In Fig. 3 we show the behavior of $\nu$ as a function of $q v_{F} / \omega$ for the case that only the $g_{2}$ scattering process is periodically modulated in time with $\bar{\rho}=-0.6$ and $\rho=0.25$ in Eq. (4) while $g_{4}=-0.4$. 


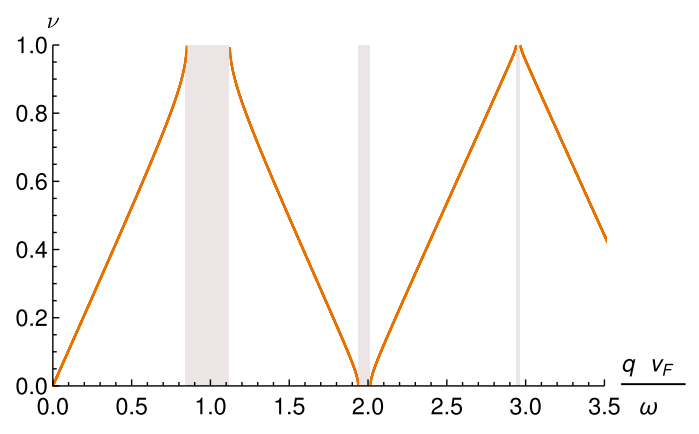

FIG. 3. Characteristic exponent $\nu$ for $g_{4}=-0.4$, while only $g_{2}(t)$ is driven with $\bar{\rho}=-0.6$ and $\rho=0.25$ in Eq. (4).

While quantitative changes compared to Fig. 2 (top) can be identified, the regions of instabilities are again found at resonant wave vectors. In Figs. 2 (top) and 3 we see that $\nu \rightarrow 1$ near the unstable regions and the ratio $c_{2} / c_{1}$ in Eq. (12) becomes singular.

To understand the physical significance of the instability regions, it is essential to consider damping. Intrinsic damping is always present in the TLL description due to higher order boson-boson interaction terms [48], which lead to a finite quasiparticle lifetime. A corresponding broadening of spectral peaks is seen numerically for finite energies and in finite systems $[77,78]$. The size of damping is not universal since it depends on microscopic details including the system size, but it can be assumed to be smaller than all other energy scales. In Ref. [75] it was shown that solutions of damped Mathieu equations become always stable for amplitudes below a given threshold. We also find that a finite lifetime $\tau_{0}$ in the form of an imaginary energy correction $\operatorname{Im} \lambda_{1}=-1 / \tau_{0}$ leads to convergence of instabilities as discussed below.

Results.-We are now in the position to calculate physical observables. The main effect of the time periodic driving is the excitation of density waves in the steady state. The number of density excitations $b_{\chi q}^{\dagger} b_{\chi q}(\chi=L$ or $R)$ in the transformed ground state $\left|u_{0}(t)\right\rangle$ is given by

$\eta_{q}=\left\langle u_{0}(t)\left|b_{\chi q}^{\dagger} b_{\chi q}\right| u_{0}(t)\right\rangle=\left\langle 0\left|\beta_{\chi q}^{\dagger} \beta_{\chi q}\right| 0\right\rangle=\left|\gamma_{2}(t)\right|^{2}$.

In Fig. 4 we plot the time average $\bar{\eta}_{q}$. For small $q$ we find that $\bar{\eta}_{q}$ approaches the static limit, but a strong divergence is observed as the instability region around $q_{\ell}$ is approached. In the inset of Fig. 4 we exemplarily show that a finite lifetime $\tau_{0}=10^{4} / v_{F} q$ turns the divergences of $\bar{\eta}_{q}$ into large maxima around $q_{\ell}$. The height of the maxima can be tuned by the product $\rho \tau_{0}$.

A universal physical picture emerges analogous to a resonance catastrophe: A finite lifetime has little effect away from resonance, but the resonance response is overwhelmingly large and proportional to $\tau_{0}$. If $q_{\ell}=\ell \omega / 2 \bar{v}<q_{c}$ is in the TLL regime, such maxima will therefore dominate the

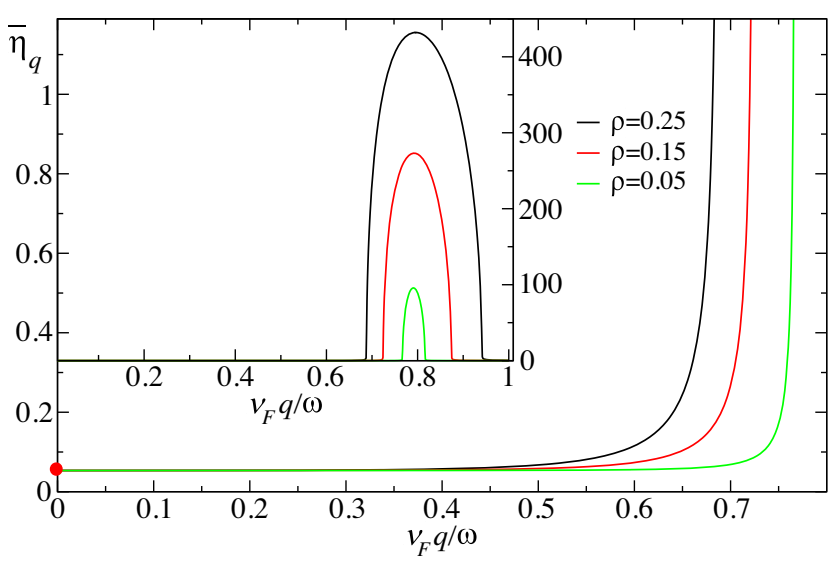

FIG. 4. Time average of $\eta_{q}(t)$, plotted as a function of $q v_{F} / \omega$ for $\bar{\rho}=-0.6$ using different amplitudes $\rho$. For $q \rightarrow 0$ the static limit $\left|\bar{\gamma}_{2}\right|^{2}=(1 / \bar{K}+\bar{K}-2) / 4$ is recovered (red dot). Inset: with finite lifetime $\tau_{0}=10^{4} / v_{F} q$ the divergent regions are turned into overwhelmingly large maxima.

correlations. We find that $q_{c} \sim \bar{v} m / 2$ is a good estimate for the cutoff.

It is well known how TLL correlations are calculated [48], which is reviewed in the Supplemental Material [73] for the example of density-density correlations [73]. An overwhelming maximum of $\eta_{q}$ will dominate the correlations and lead to long-range density order [73]

$$
\left\langle u_{0}|n(x) n(y)| u_{0}\right\rangle \propto \cos q_{\ell}(x-y) .
$$

For large driving amplitudes $\rho$ the magnitude of the induced density waves can become larger than the background density, which may lead to fragmentation into irregular density grains.

Discussion.-The three energy scales $\omega, \bar{v} q_{c}$, and $v_{F} q$ determine the behavior of the system, which undergoes three different regimes as the frequency is changed: (i) High frequencies: for $\omega \gtrsim \bar{v} q_{c}$ the instability regions are outside the TLL regime, so the physical relevant region is free of resonances. The transformation $Q$ results in a systematic change of $\eta_{q}$ shown in Fig. 4, which approaches the static limit as $q \rightarrow 0$. The famous power-law correlations [48] are corrected for intermediate distances, but the asymptotic static limit is recovered. (ii) Intermediate frequencies: as the frequency is lowered, the resonant wave numbers $q_{\ell}=\ell \omega / 2 \bar{v}$ drop below the cutoff $q_{c}$ into the TLL regime. The number of density waves $\eta_{q_{\ell}}$ becomes very large, dominating the correlations in Eq. (16). Instead of powerlaw correlations, standing density waves at wave numbers $q_{\ell}$ become stable throughout the system. (iii) Very low frequencies: for $\omega \ll \bar{v} q_{c}$ extended regions of instability will lead to a large number of excitations and heating, destroying the correlations.

Using typical experimental parameters for a $1 \mathrm{D}^{87} \mathrm{Rb}$ gas from Ref. [51] of $n=6.2 \times 10^{6} / \mathrm{m}$ and $\mathrm{mg} / \mathrm{n}=0.6$, we 
arrive at $\bar{K} \approx 4$ and a cutoff of $\omega_{c}=\bar{v} q_{c} \approx 2 \pi \times 1.4 \mathrm{kHz}$ in the middle of the trap. Driving the perpendicular confinement with a frequency of $\omega=2 \pi \times 500 \mathrm{~Hz}$ results in a resonance at $q_{1}=\omega / 2 \bar{v}=444 \times 10^{3} / \mathrm{m}$. We therefore predict a standing density wave with wavelength $\lambda=2 \pi / q_{1}$ in the $\mu \mathrm{m}$ range, which is observable in real space with optical methods or an electron beam [51,52].

The confining trapping potential leads to lower local densities $n$ near edges [51,52] and reduced velocities $v_{F}=\pi n / m$. Everywhere $n$ agrees with the local density approximation (LDA) of TLL correlations for the local trap potential $[51,52]$. The trapping potential is therefore turning into an advantage: Instead of changing the frequency $\omega$, different regimes can be reached using the changing density $n$. As a function of $n$ we know $\bar{v}=\sqrt{n g / m}$ [63-65], which in turn determines the resonant wave vectors $q_{\ell}=$ $\ell \omega \sqrt{m / n g} / 2$ and the cutoff $q_{c}=\sqrt{n g m} / 2$. Therefore, we move into the high frequency regime $q_{\ell} / q_{c} \propto \omega / n g$ as the density is lowered. Note that the density wave numbers $q_{\ell}$ increase near edges in contrast to fermionic Friedel density wave numbers, which decrease with lower densities in a trap [79]. In the proposed experiment, we therefore predict standing density waves at $\lambda \sim 14 \mu \mathrm{m}$ in the middle of the trap, which become shorter $\lambda \propto \sqrt{n}$ and weaker near the edge. It is an interesting open problem if significant corrections would be observed when going beyond the present LDA analysis for a typical trap size of $120 \mu \mathrm{m}$ in Ref. [51].

Interesting many-body density excitations have been experimentally observed in driven 1D and 2D systems [80,81]. For 1D elongated bosonic ${ }^{7} \mathrm{Li}$ gases $\mu \mathrm{m}$-sized density grains emerge at $2 \pi \times 80 \mathrm{~Hz}$ driving, which were identified as stable many-body effects [80]. Experimental images show grains that appear smaller and weaker near edges which resemble features predicted above but in a random pattern [80]. All correlations disappear for very low frequencies $\omega$. A future grain size analysis as a function of $\omega$ and $n$ may clarify if there is a relation to TLL density waves in Eq. (16).

Conclusion.-We have considered time-periodically driven interacting systems in the steady state, corresponding to generic TLL models in general or the Lieb-Liniger model in particular, which e.g., applies to 1D confined atoms in ultracold gas experiments with tunable parameters. As we have shown, this setup is one of the very rare cases where the combination of nonequilibrium steady states with many-body physics can be analyzed in great detail. In particular, we have developed a FloquetBogoliubov approach by constructing time-periodic creation and annihilation operators, which solve the eigenvalue equation for the steady state by acting on the entire Floquet space. We also identify regions in frequency-momentum space where damped resonant behavior leads to a large number of density excitations. The known static power-law correlations [48] are recovered for large distances $\gg \bar{v} / \omega$, but for frequencies below the cutoff $\bar{v} q_{c}$ characteristic density waves at integer-spaced resonant wave numbers $q_{\ell}=\ell \omega / 2 \bar{v}$ will become dominant.

We emphasize that the proposed Floquet-Bogoliubov algebra is completely general and can be used to solve any time-periodically driven model with Bogoliubov-type interactions exactly. The explicitly known transformation $Q$ maps all steady states onto a diagonal static oscillator basis for all times, which paves the way for a complete analysis of time-dependent effects in strongly interacting systems using a combination of powerful experimental, analytic, and numerical techniques.

We are thankful for support from Research Centers of the Deutsche Forschungsgemeinschaft (DFG): Projects A4 and A5 in SFB/Transregio 185: "OSCAR" and Project A10 in SFB/Transregio 173 "Spin + X."

[1] G. Jotzu, M. Messer, R. Desbuquois, M. Lebrat, T. Uehlinger, D. Greif, and T. Esslinger, Nature (London) 515, 237 (2014).

[2] M. Aidelsburger, M. Lohse, C. Schweizer, M. Atala, J. T. Barreiro, S. Nascimbène, N. R. Cooper, I. Bloch, and N. Goldman, Nat. Phys. 11, 162 (2015).

[3] P. Bordia, H. Lüschen, U. Schneider, M. Knap, and I. Bloch, Nat. Phys. 13, 460 (2017)

[4] K. Baumann, C. Guerlin, F. Brennecke, and T. Esslinger, Nature (London) 464, 1301 (2010).

[5] R. Mottl, F. Brennecke, K. Baumann, R. Landig, T. Donner, and T. Esslinger, Science 336, 1570 (2012).

[6] R. Landig, F. Brennecke, R. Mottl, T. Donner, and T. Esslinger, Nat. Commun. 6, 7046 (2015).

[7] J. Klinder, H. Keßler, M. R. Bakhtiari, M. Thorwart, and A. Hemmerich, Phys. Rev. Lett. 115, 230403 (2015).

[8] R. Landig, L. Hruby, N. Dogra, M. Landini, R. Mottl, T. Donner, and T. Esslinger, Nature (London) 532, 476 (2016).

[9] Y. Deng, J. Cheng, H. Jing, and S. Yi, Phys. Rev. Lett. 112, 143007 (2014).

[10] F. Brennecke, S. Ritter, T. Donner, and T. Esslinger, Science 322, 235 (2008).

[11] M. Kulkarni, B. Öztop, and H. E. Türeci, Phys. Rev. Lett. 111, 220408 (2013).

[12] F. Piazza and H. Ritsch, Phys. Rev. Lett. 115, 163601 (2015).

[13] D. V. Else, B. Bauer, and C. Nayak, Phys. Rev. Lett. 117, 090402 (2016).

[14] A. J. E. Kreil, H. Y. Musiienko-Shmarova, S. Eggert, A. A. Serga, B. Hillebrands, D. A. Bozhko, A. Pomyalov, and V. S. L'vov, Phys. Rev. B 100, 020406(R) (2019).

[15] Y.-J. Lin, R. L. Compton, K. Jiménez-García, J. V. Porto, and I. B. Spielman, Nature (London) 462, 628 (2009).

[16] M. Aidelsburger, M. Atala, S. Nascimbène, S. Trotzky, Y.-A. Chen, and I. Bloch, Phys. Rev. Lett. 107, 255301 (2011).

[17] Y.-J. Lin, R. L. Compton, K. Jiménez-García, W. D. Phillips, J. V. Porto, and I. B. Spielman, Nat. Phys. 7, 531 (2011). 
[18] J. Struck, M. Weinberg, C. Ölschläger, P. Windpassinger, J. Simonet, K. Sengstock, R. Höppner, P. Hauke, A. Eckardt, M. Lewenstein et al., Nat. Phys. 9, 738 (2013).

[19] P. Hauke, O. Tieleman, A. Celi, C. Ölschläger, J. Simonet, J. Struck, M. Weinberg, P. Windpassinger, K. Sengstock, M. Lewenstein et al., Phys. Rev. Lett. 109, 145301 (2012).

[20] J. Struck, C. Ölschläger, M. Weinberg, P. Hauke, J. Simonet, A. Eckardt, M. Lewenstein, K. Sengstock, and P. Windpassinger, Phys. Rev. Lett. 108, 225304 (2012).

[21] D. Thuberg, S. A. Reyes, and S. Eggert, Phys. Rev. B 93, 180301(R) (2016).

[22] S. A. Reyes, D. Thuberg, D. Pérez, C. Dauer, and S. Eggert, New J. Phys. 19, 043029 (2017).

[23] D. Thuberg, E. Muñoz, S. Eggert, and S. A. Reyes, Phys. Rev. Lett. 119, 267701 (2017).

[24] D. H. Dunlap and V.-M. Kenkre, Phys. Rev. B 34, 3625 (1986).

[25] F. Grossmann, T. Dittrich, P. Jung, and P. Hänggi, Phys. Rev. Lett. 67, 516 (1991).

[26] M. Holthaus, Phys. Rev. Lett. 69, 351 (1992).

[27] M. Grifoni and P. Hänggi, Phys. Rep. 304, 229 (1998).

[28] H. Lignier, C. Sias, D. Ciampini, Y. Singh, A. Zenesini, O. Morsch, and E. Arimondo, Phys. Rev. Lett. 99, 220403 (2007).

[29] A. Eckardt, M. Holthaus, H. Lignier, A. Zenesini, D. Ciampini, O. Morsch, and E. Arimondo, Phys. Rev. A 79, 013611 (2009).

[30] A. Rapp, X. Deng, and L. Santos, Phys. Rev. Lett. 109, 203005 (2012).

[31] T. Wang, S. Hu, S. Eggert, M. Fleischhauer, A. Pelster, and X.-F. Zhang, Phys. Rev. Research 2, 013275 (2020).

[32] V. Bretin, S. Stock, Y. Seurin, and J. Dalibard, Phys. Rev. Lett. 92, 050403 (2004).

[33] V. Schweikhard, I. Coddington, P. Engels, S. Tung, and E. A. Cornell, Phys. Rev. Lett. 93, 210403 (2004).

[34] E. R. F. Ramos, E. A. L. Henn, J. A. Seman, M. A. Caracanhas, K. M. F. Magalhães, K. Helmerson, V. I. Yukalov, and V. S. Bagnato, Phys. Rev. A 78, 063412 (2008).

[35] S. E. Pollack, D. Dries, R. G. Hulet, K. M. F. Magalhães, E. A. L. Henn, E. R. F. Ramos, M. A. Caracanhas, and V. S. Bagnato, Phys. Rev. A 81, 053627 (2010).

[36] T. Wang, X.-F. Zhang, F. E. A. dos Santos, S. Eggert, and A. Pelster, Phys. Rev. A 90, 013633 (2014).

[37] S. Greschner, L. Santos, and D. Poletti, Phys. Rev. Lett. 113, 183002 (2014).

[38] F. Meinert, M. J. Mark, K. Lauber, A. J. Daley, and H.-C. Nägerl, Phys. Rev. Lett. 116, 205301 (2016).

[39] E. Arimondo, D. Ciampini, A. Eckardt, M. Holthaus, and O. Morsch, in Advances in Atomic, Molecular, and Optical Physics, edited by P. Berman, E. Arimondo, and C. Lin (Academic Press, New York, 2012), Vol. 61, p. 515.

[40] T. Keilmann, S. Lanzmich, I. McCulloch, and M. Roncaglia, Nat. Commun. 2, 361 (2011).

[41] S. Greschner and L. Santos, Phys. Rev. Lett. 115, 053002 (2015).

[42] G. Tang, S. Eggert, and A. Pelster, New J. Phys. 17, 123016 (2015).

[43] C. Sträter, S. C. L. Srivastava, and A. Eckardt, Phys. Rev. Lett. 117, 205303 (2016).
[44] F. Lange, S. Ejima, and H. Fehske, Phys. Rev. Lett. 118, 120401 (2017).

[45] A. Eckardt, C. Weiss, and M. Holthaus, Phys. Rev. Lett. 95, 260404 (2005).

[46] A. Eckardt and E. Anisimovas, New J. Phys. 17, 093039 (2015).

[47] A. Eckardt, Rev. Mod. Phys. 89, 011004 (2017).

[48] T. Giamarchi, Quantum Physics in One Dimension (Clarendon Press, Oxford, England, 2003).

[49] T. Kinoshita, T. Wenger, and D. S. Weiss, Science 305, 1125 (2004).

[50] B. Paredes, A. Widera, V. Murg, O. Mand el, S. Fölling, I. Cirac, G. V. Shlyapnikov, T. W. Hänsch, and I. Bloch, Nature (London) 429, 277 (2004).

[51] A. Vogler, R. Labouvie, F. Stubenrauch, G. Barontini, V. Guarrera, and H. Ott, Phys. Rev. A 88, 031603(R) (2013).

[52] A. Vogler, R. Labouvie, G. Barontini, S. Eggert, V. Guarrera, and H. Ott, Phys. Rev. Lett. 113, 215301 (2014).

[53] F. Pollmann, M. Haque, and B. Dóra, Phys. Rev. B 87, 041109(R) (2013).

[54] J.-S. Bernier, R. Citro, C. Kollath, and E. Orignac, Phys. Rev. Lett. 112, 065301 (2014).

[55] P. Chudzinski and D. Schuricht, Phys. Rev. B 94, 075129 (2016).

[56] S. Pielawa, Phys. Rev. A 83, 013628 (2011).

[57] C. D. Graf, G. Weick, and E. Mariani, Europhys. Lett. 89, 40005 (2010).

[58] M. Bukov and M. Heyl, Phys. Rev. B 86, 054304 (2012).

[59] Y. Kagan and L. A. Manakova, Phys. Rev. A 80, 023625 (2009).

[60] P. Chudzinski, arXiv:1607.00995.

[61] E. H. Lieb and W. Liniger, Phys. Rev. 130, 1605 (1963).

[62] M. Olshanii, Phys. Rev. Lett. 81, 938 (1998).

[63] M. A. Cazalilla, R. Citro, T. Giamarchi, E. Orignac, and M. Rigol, Rev. Mod. Phys. 83, 1405 (2011).

[64] M. A. Cazalilla, J. Phys. B 37, S1 (2004).

[65] Z. Ristivojevic, Phys. Rev. Lett. 113, 015301 (2014).

[66] H. Ui, Prog. Theor. Phys. 44, 703 (1970).

[67] D. R. Truax, Phys. Rev. D 31, 1988 (1985).

[68] J. Garcia and R. Rossignoli, Phys. Rev. A 96, 062130 (2017).

[69] J. Sólyom, Adv. Phys. 28, 201 (1979).

[70] The applied fields are not necessarily purely sinosoidal, but $a_{0}\left[g_{2}(t)\right]$ or $a_{\perp}\left[g_{2}(t)\right]$ can be obtained exactly for a given situation and therefore also the required fields. For small amplitudes a linear expansion will generally also result in sinosoidal fields.

[71] M. Holthaus, J. Phys. B 49, 013001 (2016).

[72] While the Floquet problem always becomes static in the extended Floquet Hilbert space using frequency decomposition [46], we seek to obtain a static diagonal solution in the original Hilbert space and a transformation for all times.

[73] See Supplemental Material at http://link.aps.org/ supplemental/10.1103/PhysRevLett.126.243401 for details on the proof of the Floquet-Bogoliubov transformation, the 
relation to Floquet theory, the transformed ground state, and Mathieu functions.

[74] Here, $\nu$ is also analogous to the quasimomentum in the wellknown bandstructure of a cosine potential [71].

[75] I. Kovacic, R. Rand, and S. M. Sah, Appl. Mech. Rev. 70, 020802 (2018).

[76] W. Paul, Rev. Mod. Phys. 62, 531 (1990).

[77] I. Schneider, A. Struck, M. Bortz, and S. Eggert, Phys. Rev. Lett. 101, 206401 (2008).
[78] A. Bohrdt, K. Jägering, S. Eggert, and I. Schneider, Phys. Rev. B 98, 020402(R) (2018).

[79] S. A. Söffing, M. Bortz, and S. Eggert, Phys. Rev. A 84, 021602(R) (2011).

[80] J. H. V. Nguyen, M. C. Tsatsos, D. Luo, A. U. J. Lode, G. D. Telles, V. S. Bagnato, and R. G. Hulet, Phys. Rev. X 9, 011052 (2019).

[81] Z. Zhang, K. Yao, L. Feng, J. Hu, and C. Chin, Nat. Phys. 16, 652 (2020). 